\title{
Array analysis of reflector heterogeneity
}

\author{
J. N. Louie ${ }^{\star}$ and J. E. Vidale $\ddagger$
}

\begin{abstract}
In deep crustal reflection study, as in conventional exploration seismology, it is important to determine the geometry of the physical contrasts between rocks that cause reflections, to make reliable geologic interpretations. Fundamentally different reflecting structures produce similar signatures in stacked seismic sections. We have developed a method that uses prestack records to differentiate lateral structural variations from lateral reflectivity variations and laterally homogeneous structures. Full-wave acoustic multioffset synthetics of canonical 2-D reflector configurations, analyzed by statistically enhanced slantstack processes, show that lateral heterogeneity such as a wavy reflector can be identified from changes in slowness across a receiver array as a function of time. Application of these methods to deep crustal reflections, recorded in the Mojave Desert of southern California, identifies laterally heterogeneous midcrustal structures and is consistent with a laterally homogeneous Moho.
\end{abstract}

\section{INTRODUCTION}

The techniques presently used to analyze lithospheric seismic data have difficulty differentiating between the possible geologic interpretations of regionally-observed deep structures. While the seismic methodology has improved, both through increases in the amount and density of data collected and through consideration of reflectors more complex than flat acoustic layers, the presence of lateral heterogeneity at the target structures has confounding effects. These effects must be considered before conflicting hypotheses on the nature of lithospheric structures can be resolved.

Lateral heterogeneity has been consistently found at scales affecting seismic reflection experiments. In the deep crust, even such a prominent and widespread feature as the Moho shows evidence for heterogeneity. Braile and Chiang (1986) evaluated how the Moho responds differently to probing by refraction and reflection surveys. While the overall, long-wavelength structure of the Moho appears as a single-step discontinuity to refraction methods, Braile and Chiang (1986) proposed that a laterally heterogeneous, thinly layered structure would produce the discontinuous, multicycled fabric apparent on deep crustal stacked reflection sections.

This type of conclusion has been developed by computing essentially 1-D synthetic seismograms. Using a full 2-D solution to the acoustic wave equation, Sandmeier et al. (1987) showed that random heterogeneity in two dimensions could produce the appearance of heterogeneous layering on the reflection records. The discrimination of whether a deep structure is layered is often further confused by the lack of diffractions from segmented reflections on unmigrated stacked sections, likely due to spurious near-surface structure. Warner (1987) showed that this lack of diffractions produces the apparently overmigrated reflections often encountered in processing of deep continental data. Layering of any kind may not, however, be required to produce the multicycle reflection fabrics observed. Using 3-D ray tracing, Blundell and Raynaud (1986) showed that this fabric could be produced by a single undulating interface. Apparently, standard stacking and migration methods may not be able to distinguish the exact nature of the Moho.

Important heterogeneities other than the Moho are often imaged by lithospheric reflection surveys. For example, COCORP surveys have found bright spots interpreted to indicate midcrustal magma bodies within the Rio Grande Rift (Brown et al., 1980) and Death Valley (de Voogd et al., 1986). If these reflections are caused by magmatic features, it is still difficult to resolve whether they represent the tops of large batholiths, relatively thin horizontal sills, or small, discontinuous pillowshaped stocks or laccoliths. The various hypotheses require large differences in the volumes of magma and the nature of lithospheric extensional processes.

In the exploration of sedimentary basins, subtle lateral heterogeneities in the form of minor faults and facies changes can form the limits of a productive reservoir. Roux (1987) defined the boundaries of one such field with the amplitude and instantaneous frequency of stacked reflections. The ability to recognize

Presented at the 58th Annual International Meeting, Society of Exploration Geophysicists. Manuscript received by the Editor July 3, 1989; revised manuscript received November 6, 1990.

*Department of Geosciences, The Pennsylvania State University, University Park, PA 16802.

¥Institute of Tectonics, University of California, Santa Cruz, CA 95064.

(C) 1991 Society of Exploration Geophysicists. All rights reserved. 
these reflector lateral heterogeneities directly might independently confirm the boundaries of a reservoir and thereby speed its development.

Stacked seismic sections may not allow proper interpretation of heterogeneity. Gibson and Levander (1988) have pointed out that the stacking process, through the assumptions of 1-D geometry inherent in its derivation, can blur the distinctions between heterogeneity at the reflector and heterogeneity above the reflector. Scattering among 2-D heterogeneities will distort a deeper reflection to make it appear discontinuous, while complicating reflections from structures within the heterogeneous region. To interpret important structures such as subduction complexes and magma bodies properly, seismic acquisition and interpretation techniques must be extended to address 3-D lateral heterogeneity.

Full recognition of 3-D structures has two key elements. First, as demonstrated by Louie et al. (1988), the interpretation of seismic phases from laterally heterogeneous structures requires analysis before stacking. Stacking destroys the offset-dependent information crucial to identifying heterogeneous structure. Second, arrays of sources and receivers should be deployed and analyzed. The ability to quantify the propagation direction of arrivals both in and out of the vertical plane connecting the source and receiver would aid the characterization of lateral heterogeneities. This paper approaches characterization of heterogeneities through 2-D modeling of different types of reflector heterogeneity. Analysis of phase relations between prestack arrivals allows the distinguishing effects of each type of heterogeneity to be observed.

\section{SYNTHETICS}

We modeled the effects of 2-D heterogeneities on seismic reflection surveys via fourth-order finite-difference solutions of the acoustic wave equation. These methods are accurate and effective in considering 2-D heterogeneities (e.g., Vidale et al., 1985; Vidale, 1990), including all multiple reflections, diffractions, and surface waves.

This full-wave modeling is applied to the problems of reflector geometry discrimination. We generated synthetic shot gathers for four fundamentally different reflector configurations (Figure 1). Although any real lithospheric structure may be some combination of one or more types, synthetic data from such endmember models can be examined for the characteristic effects of each model. Thus a particular effect in a real data set indicates a reflector structure similar to that of the associated model.

Figure 1 shows four such canonical deep reflector geometries in cross-section: a simple step discontinuity; a discontinuity where the lower medium varies laterally in velocity; a reflector having sinusoidal topography; and a series of laminas. Above the boundary, the velocity is $6 \mathrm{~km} / \mathrm{s}$. Below the variable velocity reflector, the velocity varies sinusoidally between 6.5 and $9.5 \mathrm{~km} / \mathrm{s}$ (with the periodicity shown) to represent an extreme case of velocity variation in the upper mantle. In the layered case, the laminas alternate between 6 and $8 \mathrm{~km} / \mathrm{s}$. These models, depending upon their scale, could appear similar on lithospheric reflection stacked sections or give similar refraction traveltimes; yet, the presence of each structure could lead to a radically different geologic interpretation.

Multioffset finite-difference shot gathers computed for each model show reflection arrivals with ostensibly normal moveout
(Figure 2). The four models produce distinguishable multioffset records. While the first arrivals from the four models may be similar, the reflections from the heterogeneous models have additional phases, or codas, which arrive just after the primary. Moveout variations between the different models are clear on the synthetic gathers (Figure 2), but some kind of stacking technique must be employed to cull the desired signal from generally noisy lithospheric data.

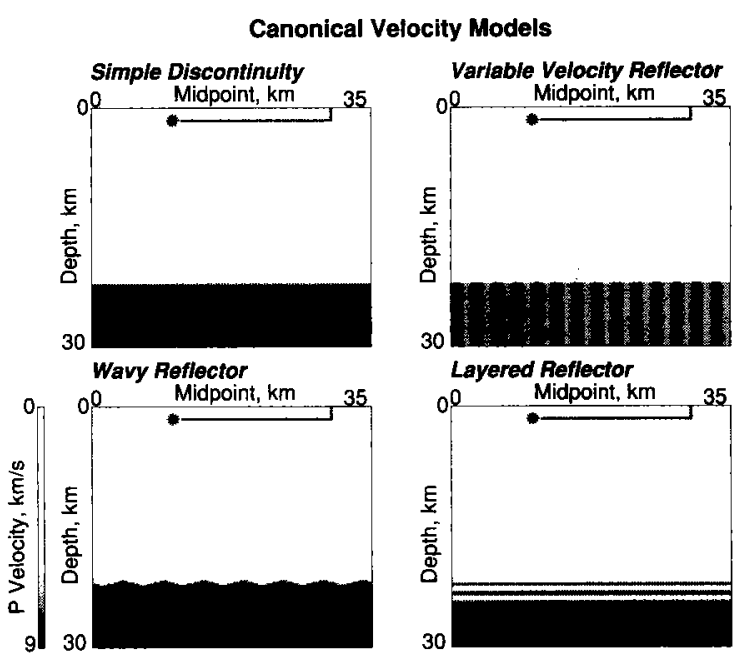

FIG. 1. Velocity cross-sections of four basic types of structural variation. The asterisks show locations of synthetic sources, which were "recorded" over the intervals spanned by the lines to the right. All sources and receivers were at the surface. Reflecting boundaries begin at $22 \mathrm{~km}$ depth.

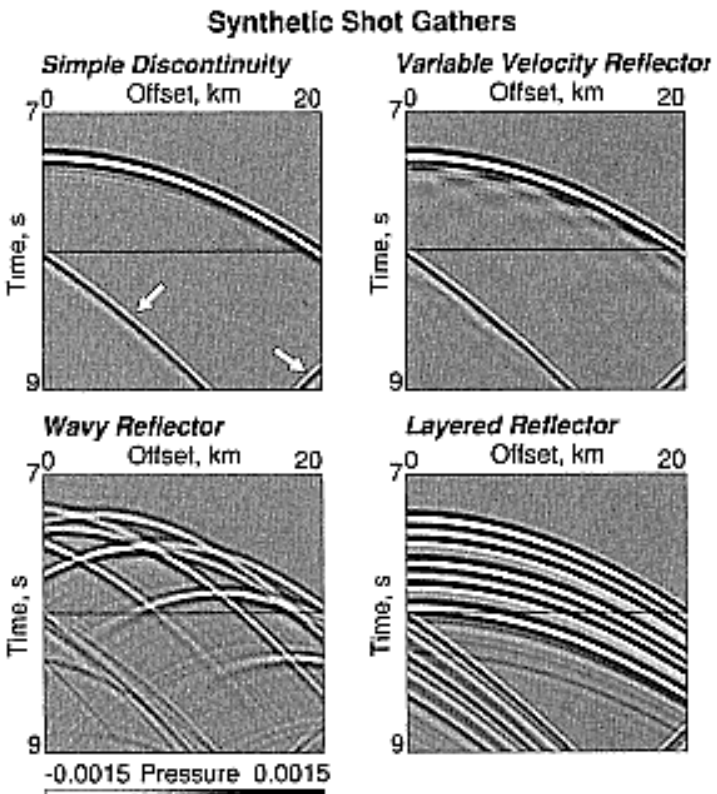

FIG. 2. Multioffset acoustic shot records computed from the models of Figure 1. Different structures produce variable moveout of phases in the codas behind the primary reflections. The arrows indicate computational artifacts reflected from the sides of the models. 
We analyze the effect of different reflector geometries on the apparent velocities of arrivals at seismic arrays with the slant stack (Schultz and Claerbout, 1978) or ray parameter-intercept time $(p-\tau)$ stack. We used a space- and time-domain slant stack that allows the analysis of discontinuous data with, for example, missing traces. Slant stacking provides methods both to estimate the ray parameter of arrivals and to evaluate the error of such determinations. We use the Bayesian statistical techniques developed in Harlan et al. (1984) to distinguish slant-stacked coherent arrivals from slant-stacked noise and artifacts.

The techniques of Harlan et al. (1984) require calculation of slant stacks of both the data and of an estimate of noise, which does not have trace-to-trace coherency. A noise gather is approximated by inverting the sign of the amplitude for a randomly chosen half of the traces in the data gather. This noise estimate is then slant stacked in the same manner as the data. Estimating noise directly from the data has the advantage of accounting for the offset-truncation artifacts of the slant-stacking process. Coherent noise will not be suppressed, but would be separated from reflections in the slant stack if it has a different moveout.

Figure 3 shows the slant stack of each synthetic gather. It also shows the slant stacks of the noise gathers estimated from the wavy and layered reflector synthetic gathers. For these examples, the traces having offsets between 14 and $20 \mathrm{~km}$ were slant stacked. The arrivals from the model reflectors begin at around
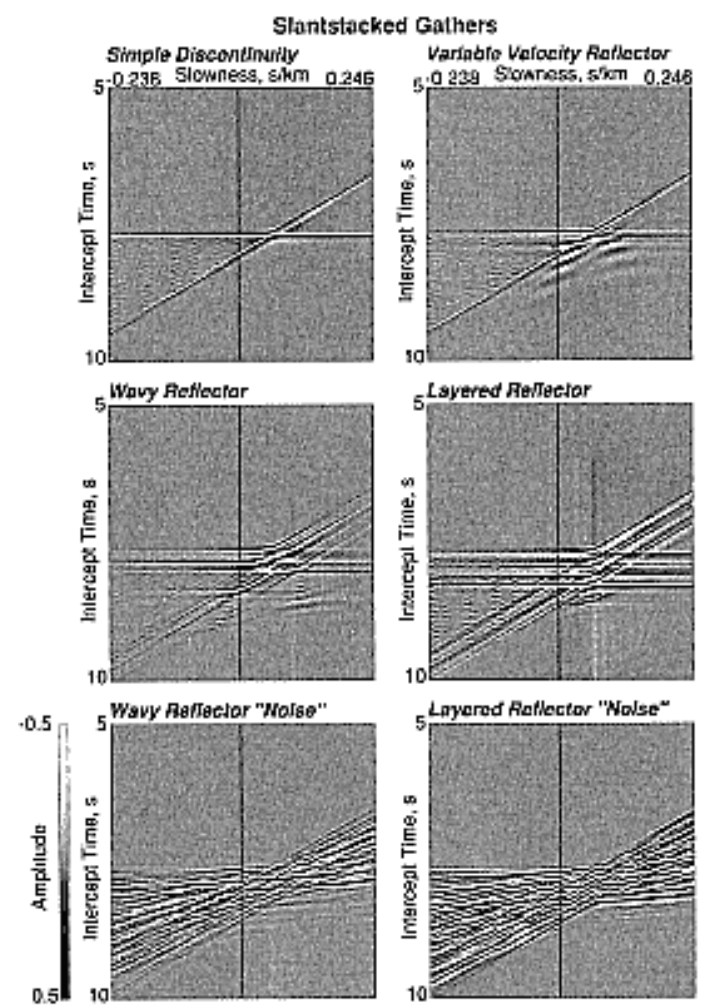

FIG. 3. Slant stack $(p-\tau)$ images computed from the $6-20 \mathrm{~km}$ offsets of each of the synthetic shot gathers of Figure 2. The primary reflections would ideally stack to a point just below and to the right of the center of each image. The linear artifacts radiating from the points are caused by the offset limits of the synthetics. The bottom two images are slant stacks of synthetics with their coherency destroyed by randomly inverting trace amplitudes. They serve to characterize the slant-stacking artifacts and are used to generate the coherency results of Figure 4.
$7.6 \mathrm{~s}$. Other offset intervals could be used as well, although a look at Figure 2 suggests that the reflection coda would be similar from any precritical offset range. The reflections from the sides of the model were muted before slant stacking.

The method of Harlan et al. [1984, equation (2)] finds the expected percentage of coherent signal present at each point of the slant stack by comparing amplitude histograms of the slant stack and of the slant-stacked noise gather. A histogram of an ideal slant stack of a gather containing only coherent signal is estimated by point-by-point division of the data histogram by the noise histogram. A minimum water level for the noise histogram was set to 0.1 percent of its maximum value to avoid unbounded division. Each set of three data, noise, and signal histograms was inspected for each model to ensure proper setting of histogram parameters and water level.

The computational cost of slant stacking the noise gathers is negligible if the procedure is concurrent with slant stacking the data. Amplitudes from data gathers can be slant stacked directly into a data stack, while the (possibly) sign-reversed amplitudes can be slant stacked using the same slownesses into a separate noise stack. Thus the only significant overhead of using Harlan et al. 's (1984) method is the storage of an additional stack.

The signal coherence of the slant stack forms an image that marks the slowness and intercept time of coherent arrivals in noisy data (Figure 4). The dark areas of the images identify the slowness and intercept time of the most energetic and coherent arrivals in the synthetic gathers. The effects of the slant stacking artifacts have been reduced.

The images in Figure 4 suggest the slowness resolution attained by the slant stack procedure. A dark area, locating the slowness of an arrival, has a width corresponding to the width of the area of higher coherence that sets it apart from the background level of expected signal. For many arrivals in Figure 4, this width is about $0.03 \mathrm{~s} / \mathrm{km}$, which is a more conservative estimate of slowness resolution than assuming resolution is limited by the

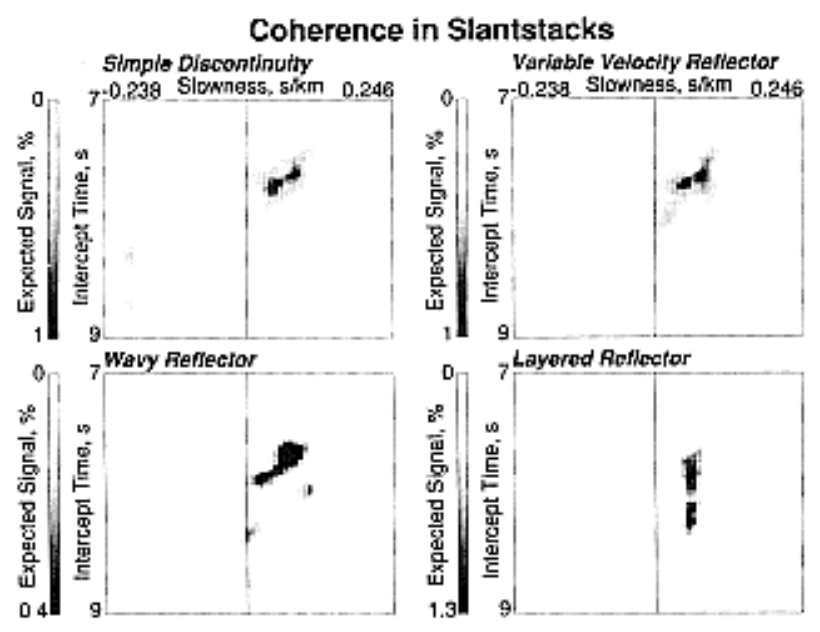

FIG. 4. Coherent signal expected at each point of a window of interest from the four slant stacks shown in Figure 3, calculated in the manner of Harlan et al. (1984). This process distinguishes reflection signals from slant-stacking artifacts. The slowness and intercept time associated with each synthetic reflection can be seen as the dark patches. Slowness resolution, estimated from the widths of the patches, is about $0.03 \mathrm{~s} / \mathrm{km}$. 
need to offset wavelets by at least half their principal period over the entire width of the receiver array. The $12 \mathrm{~Hz}$ principal frequency and $6.7 \mathrm{~km}$ array width of these synthetics imply 0.006 $\mathrm{s} / \mathrm{km}$ resolution.

In Figure 4, the simple discontinuity and the variable velocity reflector models produce arrivals that do not vary much in slowness or intercept time. The wavy reflector produces a coda of late arrivals that vary in slowness over a $0.1 \mathrm{~s} / \mathrm{km}$ range, several times larger than either estimate of slowness resolution. The late arrivals are noticeable as the dark spots below the main spot in the image. On the other hand, the layered reflector produces a sequence of arrivals that is constant in slowness relative to the $0.03 \mathrm{~s} / \mathrm{km}$ resolution.

At each intercept time, the slowness with the maximum signal expectation can be picked from the slant stack. Figure 5 plots this slowness against time for each of the synthetic gathers (top of each panel) and compares it with the maximum signal expectation (bottom of each panel). Where there are no substantial arrivals, of course, the slowness can vary wildly, often following slant-stack artifacts. Where the signal expectation is

\section{Slowness vs. Time}
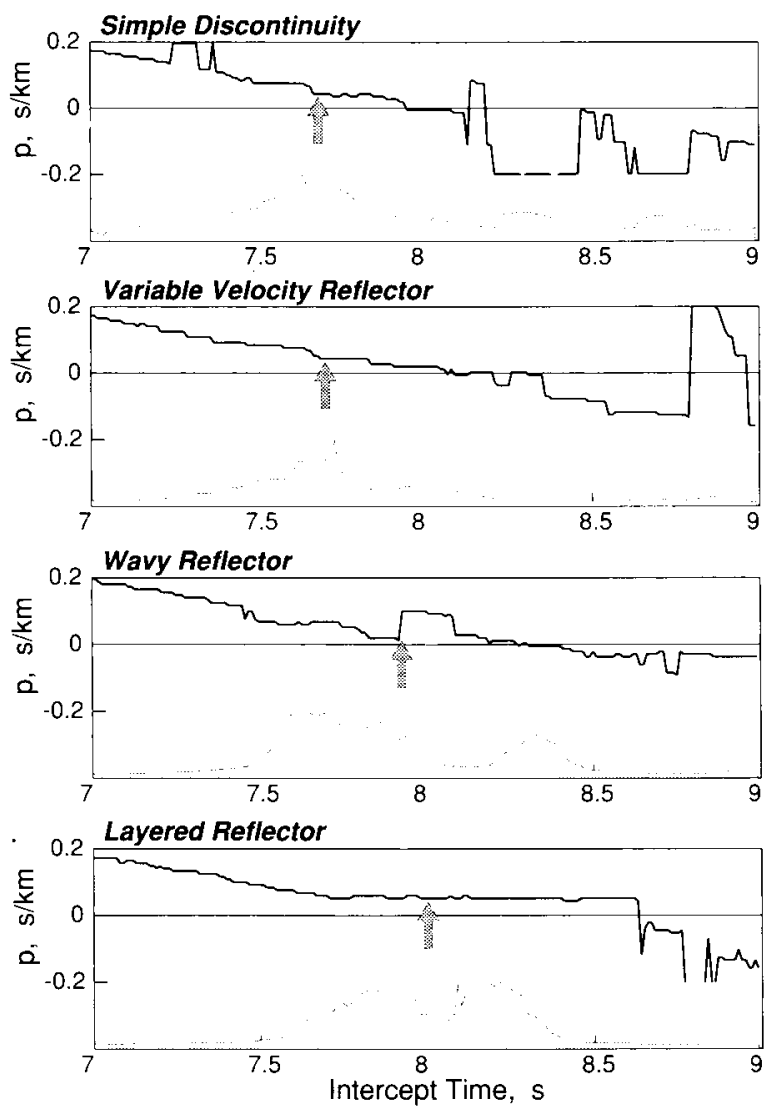

FIG. 5. Slowness versus time for the four reflector models shown in Figure 1. The upper curve identifies the slowness of the maximum expected signal at each intercept time in the images of Figure 4. It is not plotted where it exceeds the limits shown. The lower curve shows the maximum coherency for each intercept time. It peaks at coherent reflected arrivals, whose slowness variations can be identified on the upper curve. More confidence can be placed in slowness variations associated with higher coherencies and larger resolution than $0.03 \mathrm{~s} / \mathrm{km}$. The arrows indicate slowness features described in the text. larger, one can identify an arrival and find its slowness as a function of time.

Distinctions among the four model cases can be immediately drawn. The slowness characteristics of each case are most distinct in Figure 4. Figure 5 simply allows comparison of peak coherency slowness values. For both the simple and variable velocity reflectors, a steady decrease in slowness with time indicates the effect of normal moveout. For the wavy reflector, the slowness can change sharply near a strong arrival, such as at $7.95 \mathrm{~s}$ where it varies more than $0.1 \mathrm{~s} / \mathrm{km}$. Diffractions from lateral heterogeneities appear in Figure 4 as secondary peaks at a range of slownesses. The change in slowness in this case opposes the overall shifts due to normal moveout and is greater than the conservative estimate of slowness resolution. On the other hand, the reflection from the layered structure produces no resolvable variation in slowness.

The diffractions from lateral heterogeneities would often be obscured in the multioffset gathers by noise and later arrivals.

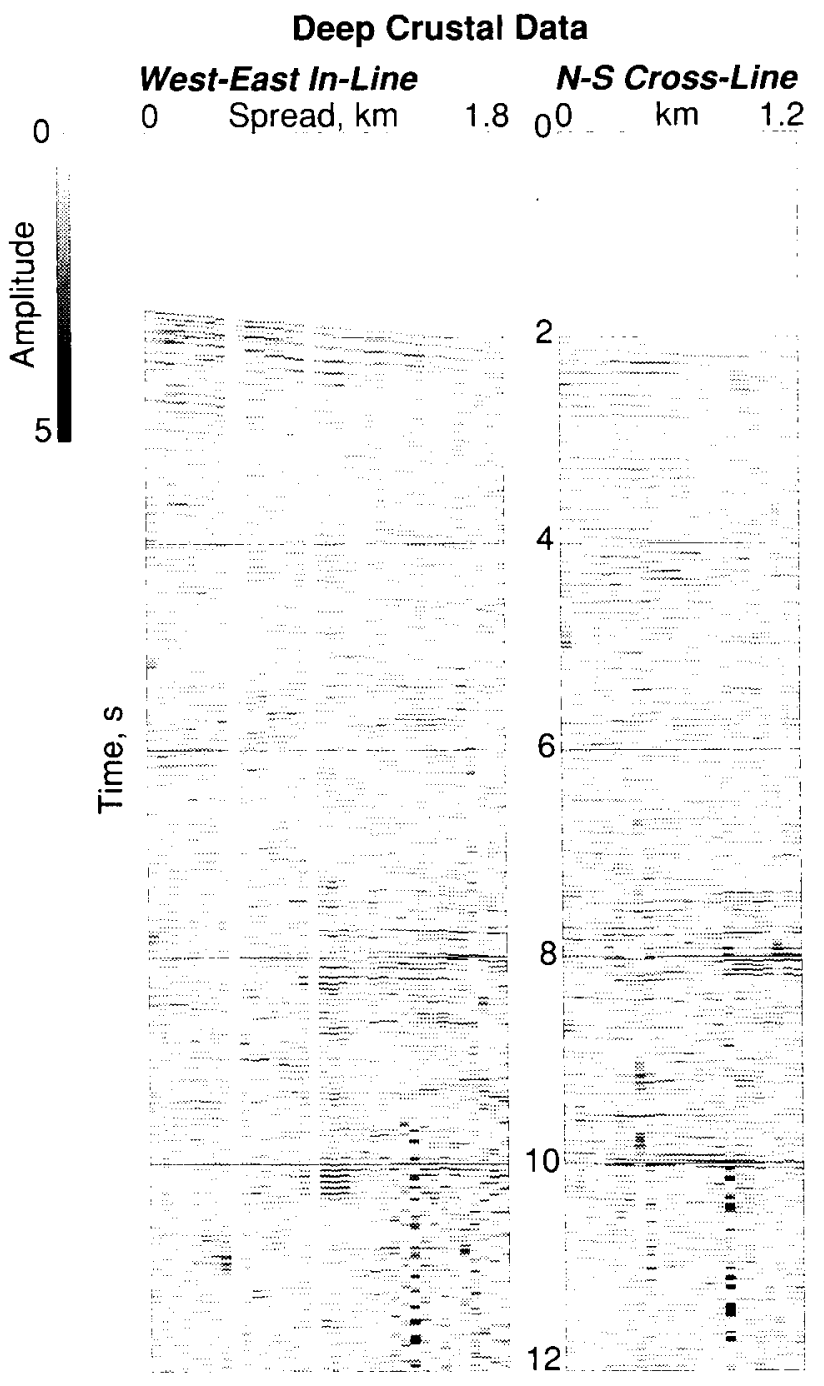

FiG. 6. Deep crustal shot gathers of two crossed receiver arrays from Dix (1965). Both arrays were located $10 \mathrm{~km}$ east of the shot. The west-east array is $1.8 \mathrm{~km}$ long and the north-south array is $1.2 \mathrm{~km}$ long. Automatic gain control was applied to balance amplitudes. Reflections arrive at $\sim 4 \mathrm{~s},-8 \mathrm{~s}$, and from the Moho at $\sim 10 \mathrm{~s}$. 
This slant-stack analysis may be able to identify such hidden slowness variations. Thus, by virtue of rapid shifts in slowness within the most coherent arrivals, reflections from structures having topographic relief on a scale greater than the seismic wavelength and the Fresnel radius may be distinguished.

Strong lateral heterogeneity above the reflector could certainly mimic heterogeneity at the reflector (Gibson and Levander, 1988). If, however, a more homogeneous structure is demonstrated at a greater depth, then the effect of strong intralayer heterogeneity can be ruled out (see the example below).

\section{DEEP CRUSTAL REFLECTION DATA}

We demonstrate the applicability of these techniques to seismic reflection receiver array data from the lithosphere with an analysis of a small data set shot in 1962 by C. H. Dix (Dix, 1965) at Soggy Lake in the Mojave Desert. Although limited in scope, this survey was carried out carefully enough to yield very clear multioffset records of deep continental reflections. The data were digitized from the survey's original magnetic drum recordings with R. Le Bras under Dix's supervision in 1983.

The original survey included three pairs of crossed lines, 1 to $2 \mathrm{~km}$ long, into which shots were made from a common source location. Data from one of these crossed arrays, recorded at an offset of $10 \mathrm{~km}$, are displayed in Figure 6 . This offset is at subcritical distance for deep reflections. Numerous arrivals can be seen on both lines, with zones of stronger events at about $4 \mathrm{~s}$ and $8 \mathrm{~s}$, and with Moho reflections arriving at about $10 \mathrm{~s}$. Given the $14 \mathrm{~Hz}$ principal frequency and 1.2 to $1.8 \mathrm{~km}$ width of the two arrays, slowness resolution can be no finer than 0.02 to $0.03 \mathrm{~s} / \mathrm{km}$.

We applied the slant-stack processes described above to these gathers. While the slant stacks are difficult to interpret for slownesses, the signal expectation images are informative (Figure 7). The darker areas indicate the time and slowness of the major

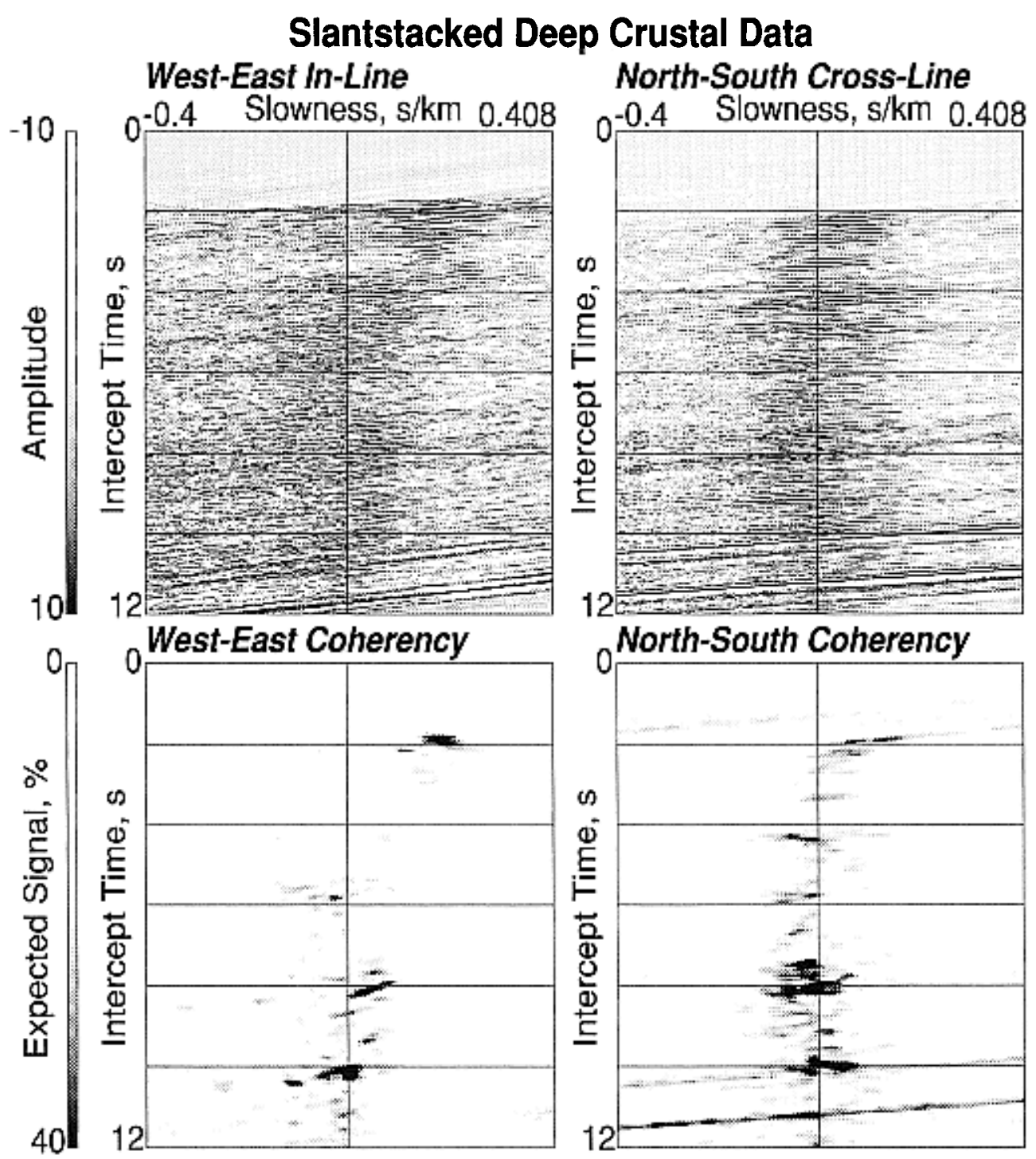

Fig. 7. Slant-stacked images of the data from Figure 6. The top two images are slant stacks of each array. The linear features are slant-stacking artifacts of the offset limits. The lower two images show the expected coherent signal at each slowness and intercept time of the corresponding slant stacks. The slowness of the first arrivals can be identified near $2 \mathrm{~s}$, while that of deep reflections can be seen down to the Moho at $10 \mathrm{~s}$. Widths of coherent arrivals in this display suggest slowness resolution near $0.03 \mathrm{~s} / \mathrm{km}$. 
arrivals. The widths of these areas suggest slowness resolution on the order of $0.03 \mathrm{~s} / \mathrm{km}$, near the limit possible for these records.

For the west-east array, the arrivals decrease in slowness after the first arrival at $2 \mathrm{~s}$, probably because for flat layers, normal moveout at a given offset decreases with increasing reflector depth. Many reflections on both arrays show slowness variability (up to a few times the level of resolution), both toward and away from the shot. The most coherent Moho reflection, at $10 \mathrm{~s}$, is near zero slowness as expected.

Dix (1965) interpreted these slowness variations in terms of dipping plane reflectors. The variability of slowness with time for these events (Figures 7 and 8 ) indicates that more information to aid discrimination between the model reflector geometries of Figure 1 may be available on these structures. Some strong arrivals show substantial variations in apparent slowness with time, such as at $4.3 \mathrm{~s}$ on the north-south array and at $8.2 \mathrm{~s}$ on the west-east array. The variations are between 0.04 and 0.05 $\mathrm{s} / \mathrm{km}$, larger than the minimum resolvable, and occur during the arrival of coherent energy. Others arrivals, such as the Moho reflection at $10 \mathrm{~s}$ on both arrays, show no slowness variation greater than the resolution minimum.

These results suggest that the $4.3 \mathrm{~s}$ (at $\sim 11 \mathrm{~km}$ depth) and $8.2 \mathrm{~s}(\sim 23 \mathrm{~km})$ reflectors are laterally complex. In addition, the lateral complexity may be limited to one dimension, since distinguishable slowness variations only appear on one of the crossed arrays for each reflection. On the other hand, the Moho in this area $(-32 \mathrm{~km}$ deep) is not distinguishable from a simple
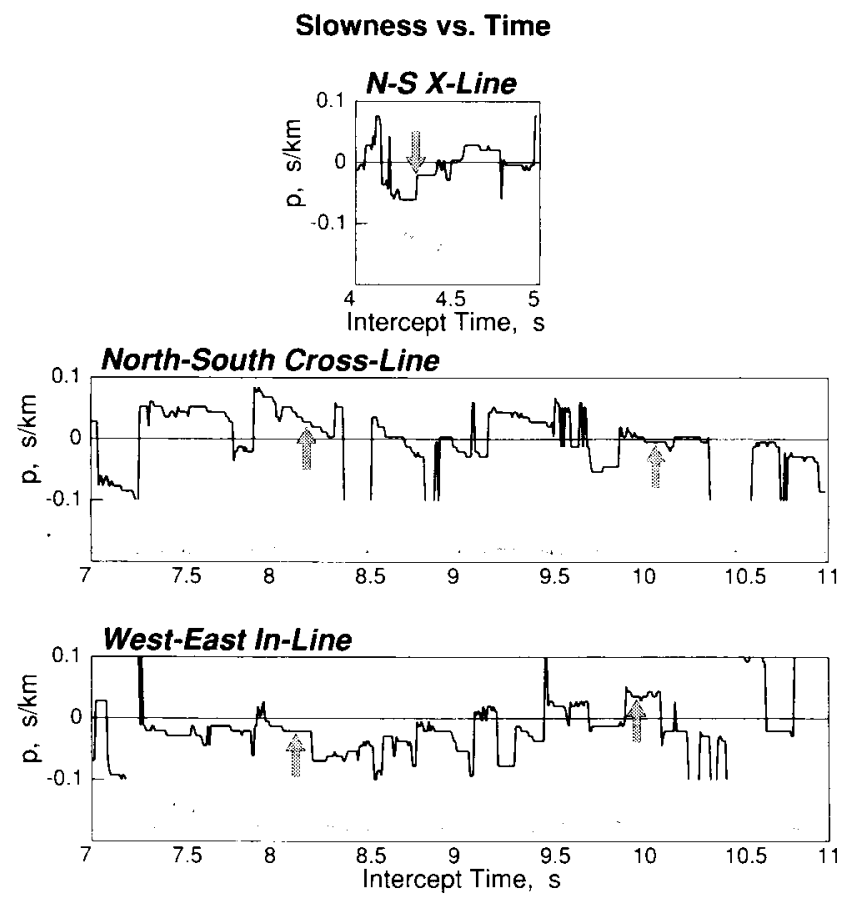

FIG. 8. Slowness versus time of reflections on the two arrays, plotted in the manner of Figure 5. The arrows identify slowness features associated with coherent reflections that characterize the lateral heterogeneity of deep structures. The Moho appears laterally homogeneous, while the shallower reflectors appear laterally heterogeneous in structure. discontinuity or laterally homogeneous laminations. Some lateral heterogeneity cannot be ruled out, since the $2.5 \mathrm{~km}$ Fresnel radius at $10 \mathrm{~s}$ time is somewhat wider than the $1.8 \mathrm{~km}$ array aperture. The reflection slowness variations, however, may well show that lateral heterogeneity is more pronounced at the shallower structures than at the Moho. More extensive study of slowness variations within data having larger ranges of offset over many more depth points would be needed to interpret the heterogeneity of these reflectors definitively.

\section{CONCLUSIONS}

Our analysis shows that modeling and slant stacking can yield new interpretations on the nature of lithospheric structures from multioffset receiver array data. In the lithosphere, the geometric configuration of reflectors is needed to constrain the possible geologic origins of deep structures. For exploration seismology, distinguishing between plane-parallel layers and subtle truncations or unconformities may be the key to locating a stratigraphically trapped reservoir. While these two types of heterogeneity may not be distinguishable in stacked sections, slant-stack analysis of prestack arrivals can provide the needed discrimination.

The development of synthetics from canonical models of reflector geometry provides criteria to identify the effects of laterally heterogeneous structure such as a wavy reflector. Statistical methods help identify the most reliable events, which can then be examined for changes in apparent slowness across a receiver array as a function of time. On a shot gather from the Mojave Desert, this procedure yielded evidence of lateral heterogeneity on shallower crustal structures. At the Moho, however, no evidence for lateral structural variations could be found.

\section{ACKNOWLEDGMENTS}

It was a great privilege to work with $\mathrm{C}$. H. Dix. We are thankful that he not only allowed us access to his data, but also willingly shared his knowledge and tremendous insight. Contribution no. 74, Charles F. Richter Seismological Laboratory. Portions of this work were supported by the Penn State Department of Geosciences, and by grants from the Amoco Foundation, the W. M. Keck Foundation, and the Institute of Tectonics.

\section{REFERENCES}

Blundell, D. J., and Raynaud, B., 1986, Modeling of lower crust reflections observed on BIRPS profiles, in Barazangi, M., and Brown, L., Eds., Reflection seismology: A global perspective: Am. Geophys. Union, Geodyamics Series, 13, 287-295.

Braile, L. W., and Chiang, C. S., 1986, The continental Mohorovicic discontinuity: results from near-vertical and wide-angle seismic reflection studies, in Barazangi, M., and Brown, L., Eds., Reflection seismology: A global perspective: Am. Geophys. Union, Geodynamics Series, 13, 257-272.

Brown, L. D., Chapin, C. E., Sanford, A. R., Kaufman, S., and Oliver, J. E., 1980, Deep structure of the Rio Grande Rift from seismic reflection profiling: J. Geophys. Res., 85, 4773-4800.

de Voogd, B., Serpa, L., Brown, L., Hauser, E., Kaufman, S., Oliver, J., Troxel, B. W., Willeman, J., and Wright, L. A., 1986, Death Valley bright spot: A miderustal magma body in the southern Great Basin, California: Geology, 14, 64-67.

Dix, C. H., 1965, Reflection seismic crustal studies: Geophysics, 30, 1068-1084. 
Gibson, B. S., and Levander, A. R., 1988, Modeling and processing of scattered waves in seismic reflection surveys: Geophysics, 53, 466-478.

Harlan, W. S., Claerbout, J. F., and Rocca, F., 1984, Signal-noise separation and velocity estimation: Geophysics, 49, 1869-1880.

Louie, J. N., Clayton, R. W., and Le Bras, R. J., 1988, Three-dimensional imaging of steeply dipping structure near the San Andreas fault, Parkfield, California: Geophysics, 53, 176-185.

Roux, R. M., 1987, Ship Shoal Block 91 field, offshore Louisiana: Discovery and development of a stratigraphic field: Geophysics, 52, $1466-1472$.

Sandmeier, K. -J., Walde, W., and Wenzel, F., 1987, Physical properties and structure of the lower crust revealed by one- and two-dimensional modeling: Geophys. J. Roy. Astron. Soc., 89, 339-344.

Schultz, P. S., and Claerbout, J. F., 1978, Velocity estimation and downward continuation by wavefront synthesis: Geophysics, 43 , 691-714.

Vidale, J. E., 1990, Comment on a comparison of finite-difference and Fourier method calculations of synthetic seismograms by C. R. Daudt et al.: Bull. Seis. Soc. Am., 80, 493-495.

Vidale, J. E., Helmberger, D. V., and Clayton, R. W., 1985, Finitedifference seismograms for $S H$ waves: Bull. Seis. Soc. Am., 75, $1765-1782$.

Warner, M., 1987, Migration - Why doesn't it work for deep continental data?: Geophys. J. Roy. Astr. Soc., 89, 21-26. 